\title{
Changes in Magnetic Parameters of Rocks Under Pressure Conditions of the Earth's Crust: Relationship to Magnetic Mineralogy
}

\author{
A. Kapička, E. Petrovský, F. Hrouda*, and J. Pohl ${ }^{\S}$ \\ Geophysical Institute, Acad. Sci. Czech Republic, Boči II/1401, 14131 Prague 4, Czech Republic \\ *AGICO, Jecná 29a, 61246 Brno, Czech Republic \\ Institut für Allgemeine und Angewandte Geophysik, Universität München, Theresienstr. 41, 80333 München, Germany
}

\begin{abstract}
Magnetic susceptibility and its anisotropy of rocks from depths down to $8081 \mathrm{~m}$ were investigated in dependence of directional stress. Dominant ferrimegnetic minerals were determined using thermomagnetic analysis of magnetic susceptibility. Depth layers, exhibiting unstable behaviour of the studied magnetic parameters under stresses corresponding to actual in situ conditions, were determined and discussed. Rock samples from these strata contain primarily magnetite or mixture of two pyrrhotite phases.

[magnetic susceptibility of rocks, AMS, deviatoric stress, magnetic mineralogy]
\end{abstract}

\section{Introduction}

Actual magnetic parameters of rocks of the Earth's crust depend strongly on physical conditions present at the depth of their burial. Since rocks are placed in stress and temperature fields significantly different from those on the Earth's surface, their in situ magnetic parameters can differ from those measured in laboratory under normal atmospheric conditions. However, knowledge of the actual values of magnetic parameters, in particular magnetic susceptibility and its anisotropy and remanent magnetization, is of great importance for several geophysical applications. It can be used in e.g. interpreting the observed local magnetic anomalies generated by sources in various depths [1].

Temperature represents a crucial parameter affecting magnetic behaviour of rocks in deeper layers, where it approaches the Curie (or Néel) temperature of main ferrimagnetic minerals. Considering the actual temperature gradient in the Earth's crust, this parameter becomes dominant in depths below $7-8 \mathrm{~km}$. On the other side, magnetic properties of rocks placed in the upper part are controlled by external stress.

Laboratory investigations of magnetic parameters of rocks, corresponding to actual conditions of the Earth's crust, require special pressure experiments. Pressure modelling has to take into account specific effects of different stress fields (homogeneous and/or inhomogeneous) on magnetic parameters as well as actual distribution of stress in different depths. Laboratory design of actual stress conditions is however always simplified. Various magnetic properties of rocks were investigated earlier under conditions of pure hydrostatic or pure uniaxial external pressure, either (e.g. [2] or [3], respectively). However, other experiments proved that extent of changes in principal magnetic parameters is clearly dominated by magnitude of deviatoric stress (e.g. [4]). This conclusion, apart from experiments supported also by theory of piezomagnetic phenomena, complicates modelling of magnetic properties corresponding to conditions of the Earth's crust, contrary to other physical parameters (e.g. elastic), which primarily depend upon hydrostatic pressure [5] and can be easily modelled in laboratory on the basis of theoretical estimate of pressure in dependence of depth. In case of magnetic parameters, values of actual deviatoric stresses in different depths are necessary prerequisite. Accordingly, laboratory modelling can be carried out, with sufficient reliability, on samples from deep boreholes where stress field was measured.

In this study, rock samples from an ultradeep KTB borehole (Bavaria, Germany) from depths down to $8081 \mathrm{~m}$ were used to examine magnetic susceptibility and its anisotropy under pressures corresponding to actual conditions of the borehole. Data on stress field within the borehole, measured using various methods, were used. Stress field is characterised by 3 main components: maximum horizontal, minimum horizontal and vertical [6]. Values of deviatoric stresses served as basis for our pressure experiments. Main magnetic carriers in samples were determined by thermomagnetic analysis of magnetic susceptibility. As a result, depth profiles of magnetic parameters were determined, with emphasise being put on layers showing pressure-unstable magnetic behaviour and its links with magnetic mineralogy.

\section{Samples and experiment}

A set of 50 samples from KTB borecores (both pilot and main borehole) were studied, covering the whole depth range of the KTB borehole down to $8081 \mathrm{~m}$ and representing main lithologic units (gneiss, metabasites). Detailed description of samples and their basic magnetic characteristics are presented in [7]. Magnetic susceptibility and its anisotropy under uniaxial pressure up to $60 \mathrm{MPa}$ was measured using a special chamber in large pick-up coils of KLY-2 Kappabridge (using alternating magnetising field of $300 \mathrm{kA} / \mathrm{m}$ ). The samples were of cylindrical shape, with diameter and height of 8 and $10 \mathrm{~mm}$, respectively. Anisotropy of magnetic susceptibility was evaluated following measurements of 15 directional values of susceptibility, measured at the same pressure. In addition, pressure dependence of mean susceptibility $\kappa_{\text {mean }}$, principal directional parameters $\kappa_{\mathrm{par}}$ and $\kappa_{\mathrm{per}}$ were evaluated. Based on the latter two parameters, coefficients of stress sensitivity $\beta_{\mathrm{par}}$ and $\beta_{\text {per }}$ can be calculated [8], which can be used in determining the stability of magnetic behaviour with respect to the stress applied.

Thermomagnetic analysis of magnetic susceptibility was carried out on the basis of measurements of magnetic susceptibility in dependence of temperature (up to $700^{\circ} \mathrm{C}$ ) using a CS-2 apparatus attached to the KLY-2 Kappabridge [9]. Carriers of the anisotropy of magnetic susceptibility were 


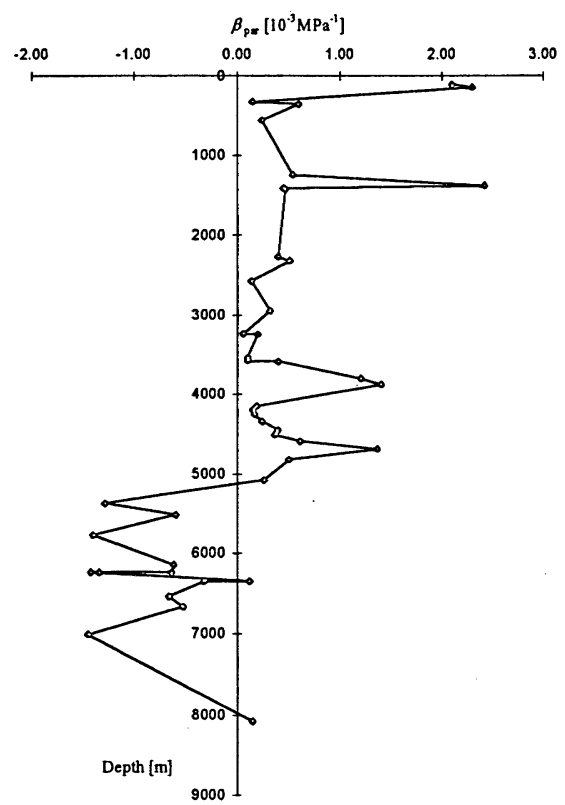

Fig. 1. Changes in stress sensitivity coefficient $\beta_{\text {par }}$ of magnetic susceptibility with depth.

determined using a method based on comparison of anisotropy of magnetic susceptibility with that of isothermal remanent magnetization, measured using spinner magnetometer JR-5.

\section{Results and discussion}

Coefficient $\beta_{\mathrm{par}}$ of stress sensitivity for the whole depth profile is depicted in Fig. 1, clearly showing great variability of the values. Significant part of the profile is characterised by comparatively low sensitivity $<0.6 \times 10^{-3} \mathrm{MPa}^{-1}$. In this case, both mean magnetic susceptibility and its anisotropy are relatively pressure-independent and rather high deviatoric stress, present in the Earth's crust, has basically negligible effect on the magnetic parameters studied. Remarkably higher values of the stress sensitivity coefficients $\left(1.2-2.4 \times 10^{-3} \mathrm{MPa}^{-1}\right)$ were found in samples from distinct depths, most significantly being expressed in depths of 105-141, about 1381, 3790-3873 and about 4686 meters. Actual stress field in these depths modifies to a great extent magnetic anisotropy of rocks. For instance, stress in depths below $4000 \mathrm{~m}$ is sufficient to reorientate completely the susceptibility ellipsoid [7]. Extraordinary values of the stress sensitivity coefficient were found in rocks from depths between 5380 and 6546 meters. In addition to high absolute values of stress sensitivity coefficient $\beta_{\mathrm{par}}\left(>1 \times 10^{-3} \mathrm{MPa}^{-1}\right)$, negative character of this coefficient was found, resulting from significantly unstable behaviour of mean magnetic susceptibility under uniaxial pressure [7]. This effect is reflected by increased values of all directional susceptibilities.

Investigation of magnetic mineralogy within the KTB borehole was carried out in order to meet the following two aims. Firstly, dominant carriers of magnetic anisotropy were determined by comparing anisotropy of magnetic susceptibility
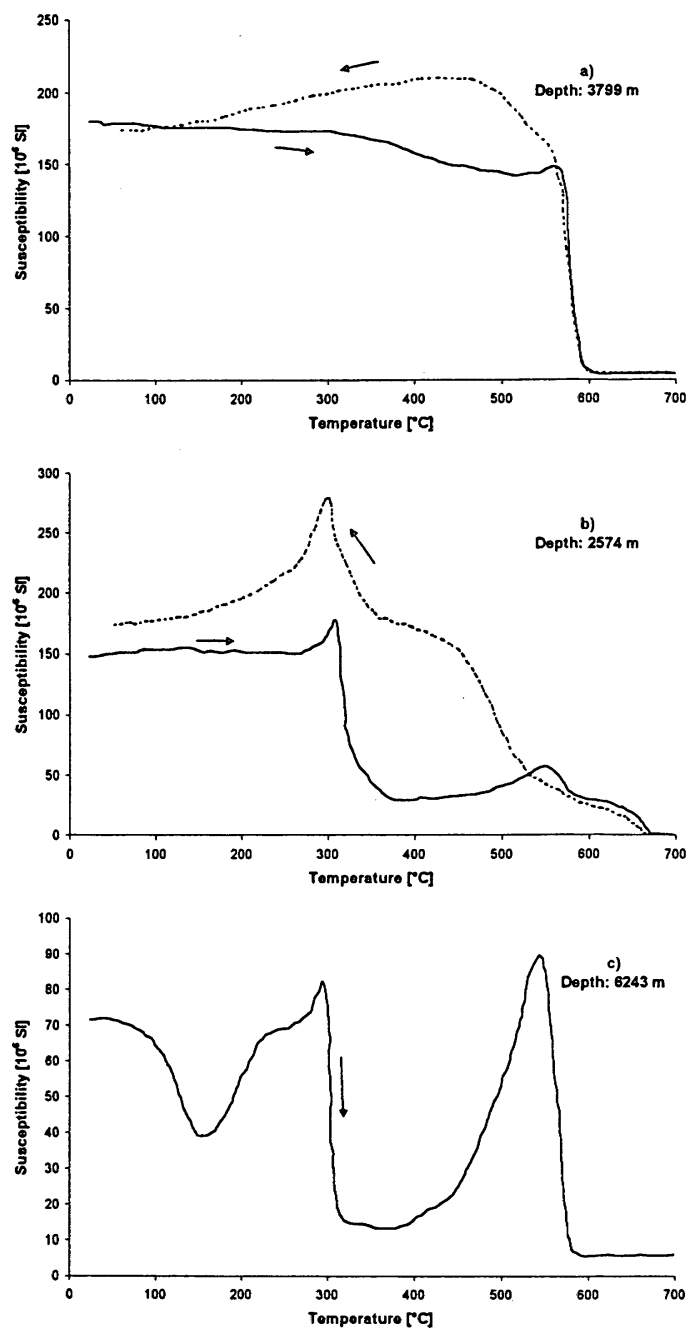

Fig. 2. Typical temperature dependence of magnetic susceptibility of KTB rock samples, used for thermomagnetic analysis of ferrimagnetic minerals.

with anisotropy of isothermal remanent magnetization (acquired in different magnetic fields). In almost all of the samples, coincidence of principal axes of the two anisotropies was found. Accordingly, anisotropy of magnetic susceptibility is predominantly controlled by ferrimagnetic minerals. This finding is of great importance for our pressure modelling due to the fact, that (despite paramagnetic minerals) only anisotropy carried by ferrimagnetics can be affected by external pressure. It is therefore evident that different values of stress sensitivity coefficients (Fig. 1) are directly linked to dominant ferrimagnetic minerals present in the samples. Reliable determination of these minerals represent the second main purpose of magnetic mineralogy.

Dominant ferrimagnetic minerals were identified on the basis of temperature dependence of magnetic susceptibility. In 
Table 1. Coefficients of stress sensitivity of KTB rocks containing different magnetic phases.

\begin{tabular}{lll}
\hline \multicolumn{1}{c}{ Magnetic phase } & $\beta_{\text {par }}\left[10^{-3} \mathrm{MPa}^{-1}\right]$ & $\beta_{\text {per }}\left[10^{-3} \mathrm{MPa}^{-1}\right]$ \\
\hline $\mathrm{Fe}_{3} \mathrm{O}_{4}$ & $1.20-2.42$ & $0.11-0.97$ \\
$\mathrm{Fe}_{7} \mathrm{~S}_{8}$ & $0.10-0.54$ & $0.11-0.64$ \\
$\mathrm{Fe}_{7} \mathrm{~S}_{8}+\mathrm{Fe}_{9} \mathrm{~S}_{10}$ & $0.31-1.45$ & $0.64-1.78$ \\
\hline
\end{tabular}

principle, three main types of thermomagnetic curves were observed, as shown in Fig. 2. Samples, showing the most pressure-unstable behaviour, contain mainly magnetite $\mathrm{Fe}_{3} \mathrm{O}_{4}$ (curve a). The other two types of samples contained mainly pyrrhotite. However, two different types of curves could be observed in this case. Firstly, a continuous increase in susceptibility at temperatures up to $300-320^{\circ} \mathrm{C}$ indicates presence of ferrimagnetic (monoclinic) pyrrhotite $\mathrm{Fe}_{7} \mathrm{~S}_{8}$ (curve b), which was found as dominant mineral in samples showing pressure-stable behaviour, characterised by low values of stress sensitivity coefficient $\beta_{\mathrm{par}}$. Finally, significant increase in susceptibility at temperatures of about $220-230^{\circ} \mathrm{C}$, followed by moderate increase up to $300-320^{\circ} \mathrm{C}$, reflects, in addition to the ferrimagnetic pyrrhotite, also presence of antiferromagnetic (hexagonal) phase $\mathrm{Fe}_{9} \mathrm{~S}_{10}$. Mixture of the two pyrrhotite phases was only found in samples from depths between 5380 and $6546 \mathrm{~m}$, showing comparatively high sensitivity to external pressure.

Actual values of magnetic susceptibility (and its anisotropy) in deeper parts of the Earth's crust are basically determined by dominant ferrimagnetic mineral present and magnitude of deviatoric stress component. Correlation of magnetic mineralogy with experimentally acquired coefficients of stress sensitivity yields rather good classification of the KTB rocks according to the contents of dominant ferrimagnetic mineral (Table 1). Since the increase of deviatoric stress with increasing depth can be more or less considered continuous (Fig. 3), actual in situ magnetic parameters are controlled by magnetic mineralogy. Pressure-unstable depth layers, determined on the basis of this classification, are shown in Fig. 3. However, samples from the upper part of the borehole (down to $3000 \mathrm{~m}$ ) exhibited rather stable behaviour of magnetic parameters upon pressure treatment of low deviatoric stress (10-20 MPa), as shown in $[10,11]$. Contrary to that, samples from depth of $4686 \mathrm{~m}(\mathrm{dS}>\mathrm{MPa})$ showed $10 \%$ higher degree of anisotropy and direction of minimum susceptibility completely biased to horizontal plane [7].

\section{Conclusions}

Results acquired using laboratory pressure experiments and modelling are necessary in order to establish actual values of magnetic parameters of rocks in the Earth's crust. In this study, pressure-induced stability of magnetic susceptibility and its anisotropy of rocks from ultradeep KTB borehole is reported and links to magnetic mineralogy discussed. We can conclude that this stability is affected by the two following factors: magnetic minerals present and magnitude of external deviatoric stress acting in the depth of the rock burial. Magnetic mineralogy seems to be dominant and divides the depth profile down to $8 \mathrm{~km}$ to sections characterised by magnetic parameters stable and unstable with respect to in situ stress conditions. The latter factor has to be considered only in rocks showing

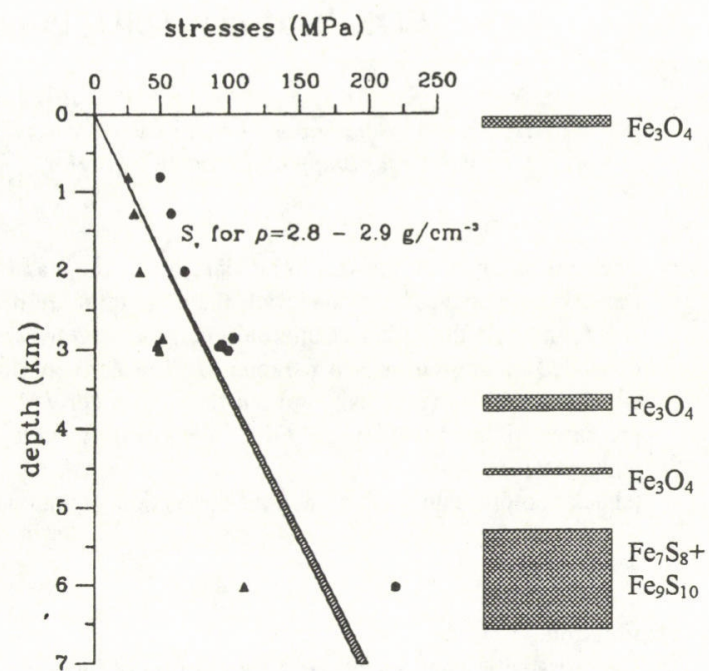

Fig. 3. Principal stress profiles to $6 \mathrm{~km}$ depth in the KTB boreholes. and $\boldsymbol{\Delta}$ represent major and minor horizontal stresses, respectively, $S_{V}$ is vertical overbunden stress (after [6]). Layers showing the found pressure-induced unstable magnetic parameters are indicated.

pressure-unstable behaviour in the above-mentioned sense. In case of KTB borehole, several pressure-unstable layers can be identified. Significant pressure-induced increase in magnetic susceptibility was observed in rocks from a wide depth range between 5380 and $6546 \mathrm{~m}$. This increase can even bring about a pressure-induced local magnetic anomaly in the Earth's crust. Despite the fact that our study is limited to the KTB borehole, conclusions on the relationship between magnetic mineralogy and pressure-stability of magnetic parameters can be applied in general consequences.

\section{Acknowledgements}

This study was supported by a Grant Agency of the Czech Republic through a grant No.205/93/0414.

\section{References}

[1] F. Fiedberg, F. Kuhnke, Scientific Drilling, 4, 139 (1994).

[2] G. J. Borradaile, M. Jackson, Phys. Earth Planet. Inter., 77, 315 (1993).

[3] A. Kapička, Phys. Earth Planet. Inter., 51, 349 (1988).

[4] A. Kapička, Phys. Earth Planet. Inter., 63, 78 (1990).

[5] T. Chlupác, Z. Pros, E. Jahns, S. Siegesmund, Proc. XXXII EHPRG Meeting, 210, Brno, 1994.

[6] F. Rummel, M. L. Zoback, KTB-Report, 93-2, 191, Hannover, 1993.

[7] A. Kapička, E. Petrovský, J. Pohl, Phys. Chem. Earth, (1997, in print).

[8] T. Nagata, Tectonophysics, 9, 167 (1970).

[9] F. Hrouda, Geophys. J. Inter., 118, 604 (1993).

[10] A. Zhang, H. Berckhemer, K. Wolter, KTB-Report, 90-8, F1-F21, Hannover, 1990.

[11] A. Zhang, K. Wolter, H. Berckhemer, Scientific Drilling, 1, 115 (1989). 